\title{
"Vous êtes toujours là? Ma souris m'a lâché » : cris, chuchotements et objets de l'écrivain dans le roman Vacuum de Christian Mistral
}

\author{
Diana Buglea \\ Huron at Western University \\ Canada
}

\section{Vacuum, la fin d'un cycle}

Portant dans l'univers de l'écriture différents chapeaux, Christian Mistral - poète, romancier, parolier, bloggeur - cultive l'image d'un homme de plume cocasse, marginal, habité par le syndrome de l'imposture. Dès son poème de jeunesse, "Saint-Denis ", il donne à lire le portrait d'un "Monarque du boulevard, / Bouffon de bohème » (Fontes 45), alors que ses romans nous révèlent des personnages pour lesquels écrire un livre « c'est juste du recyclage » ou " de la récupération de fantasmes » (Vautour 42). Au-delà de l'œuvre, sur son site web, " [r]éalisé avec le concours amusé de Christian Mistral », celui-ci avertit les lecteurs qui s'apprêtent à accéder à la page des entretiens ${ }^{1}$ qu'il est inutile de s'y attendre à un exercice de vérité : " [à] noter qu'ici, Mistral n'écrit pas, ce qui veut dire qu'il ment comme il respire, à vous en arracher les dents » (Mistral, « Présentation »).

Tout comme ses entretiens, les romans du cycle « Vortex Violet ${ }^{2}$, à savoir Vamp (1988), Vautour (1990), Valium (2000) et Vacuum (2003), invitent chacun à réfléchir à la relation qui unit l'auteur à son œuvre, d'autant plus qu'ils donnent à lire une interrogation persistante du passé de l'écrivain et des clins d'œil comme celui de la quatrième de couverture de Valium : « [i]l en a fait une œuvre qui l'a projeté bien audelà de lui-même. Une vie revue et corrigée ". Il s'en dégage aussi, au sein même du discours fictionnel, l'image d'un « intrus » (Vautour 96) voué à " clopiner sur les mesas artificielles » en attendant qu'il trouve « sa propre danse » (Valium 220). Ce portrait sans fard prendra de l'ampleur dans l'entreprise autofictionnelle de Vacuum. Ici, le narrateur, qui se nomme Christian Mistral, nous confie ses rêves, ses réflexions, ses aventures, autant que les affres du métier. Cependant, cette fois-ci, le " grand fabulant » (Vacuum 253) qui occupe la scène s'exprime par le biais de la notation journalière. À la suite de Valium, découpé en cinquante séquences narratives numérotées, Vacuum se donne à lire comme un texte encore plus fragmenté, fait d'entrées quotidiennes hétérogènes - "lambeaux " textuels livrés sous l'étiquette de roman. C'est sous le signe du journal que s'achève un cycle, et au lecteur de prêter cette fois-ci l'oreille aux cris et chuchotements d'un écrivain qui n'arrive pas à faire avancer son livre, Origines, et au crépitement du clavier qui a remplacé la machine à écrire.

Puisque Vacuum est avant tout ce texte écrit explicitement au clavier, nous avons choisi d'aborder en premier la question du rapport de l'écrivain au dispositif informatique, l'engouement de celui-ci pour l'ordinateur et ses possibilités, tout en montrant que cet outil, grâce auquel voit le jour son journal, est un objet qui incite le scripteur à réfléchir sur lui-même en puisant dans l'imaginaire numérique. ${ }^{3}$ Dans un deuxième temps, sachant que Vacuum a connu les hauts et les bas d'un processus d'autopublication ${ }^{4}$, nous allons souligner la dimension dialogique du journal intime ainsi exhibé qui n'est plus cet objet du repli sur soi, du retrait, selon la tradition, mais une sorte d'objet de correspondance avec ses cyberlecteurs. En lien avec cette problématique, nous allons suivre le devenir du journal alors que se profile à l'horizon l'objet-livre, tout en précisant la relation de l'écrivain avec le dictionnaire - objet-fétiche? - et la façon dont il manifeste son amour des mots.

\section{L'écrivain au clavier}

Vacuum fait état du quotidien d'un écrivain qui, dans son studio, qu'il décrit à plusieurs reprises, s'amuse à rêver d'écrire " l'histoire d'un homme qui aime la littérature, la bière et la liberté » (226), mais se trouve souvent saisi d'une évidence amère : « [d]'auteur prolifique, je suis devenu celui qui livre au comptegouttes, et le mieux est que j'intègre cette conception de moi-même le plus totalement possible " (64). Le journal qu'il tient dans son "Bunker », sa " boîte à vivre » (42) - espace minimaliste - est une sorte de refuge en temps de crise, un pari autobiographique faute de mieux. Or le pari du journal est facile, comme le rappelle Philippe Lejeune, car il s'agit de témoigner de son existence dans un cadre relativement dénué de contraintes: « c'est tout simple, on a du papier, ou son ordinateur, on met la date, on écrit ce qu'on fait, ce qu'on sent, ce qu'on pense » («Composer un journal»). Tout un chacun saurait en tenir un. Et au diariste de Vacuum d'embrasser cette simplicité jusque dans l'évocation de son chez-soi : 
Des courants s'y assemblent, un faisceau de forces, une gerbe d'énergies. L'hydro-électricité circule de la baie James jusqu'aux prises encastrées dans les murs. J'y branche deux ventilateurs, cinq ampoules, un tube au néon, une télé, un magnétoscope, un ordinateur, un moniteur, un modem, une imprimante, deux hauts-parleurs, un grille-pain, un poêle, un frigo, une cafetière espresso, un aspirateur et deux cells', plus le câble. (42)

Penchons-nous davantage sur cette image du diariste casanier, avec ses objets de toute sorte, avant de découvrir l'écrivain au-delà du repli domestique visible dans plusieurs entrées. Serait-il utile, par ailleurs, de lire entre les lignes de cet éloge de l'énergie tacite des choses le contrepoint du manque de souffle de celui qui, livrant des mots " au compte-goutte ", se donne la peine de les contempler ? Sans doute est-il intéressant de remarquer dans cette description minimaliste l'absence d'allusion à l'écriture : l'ordinateur et l'imprimante - outils de l'écrivain, mais aussi de tout un chacun - sont ici des objets quelconques parmi d'autres appareils possédés, et celui qui en parle choisit de le faire en simple utilisateur. Nous pouvons citer, dans la même veine, une autre entrée où s'affiche une telle tranquillité du foyer avec, cette fois-ci, la présence des livres (et du possessif) : « [d]'ici, de mon bureau, j'embrasse tout mon minuscule et précieux univers. Ma vue, ma tévé, mon lit, mon frigidaire, ma bibliothèque et mon Kevin endormi sur le sofa dans un drap léger de coton propre, un micro-ventilo calculé pour lui flatter la couenne » (111). II arrive aussi, finalement, que le décor intime communique une urgence transgressive qui trahit la griffe de l'écrivain. Comment d'ailleurs ne pas remarquer, par exemple, le travail esthétique à l'œuvre dans cette notation relative à un changement de comportement - une " mise en boite » du je ? Comment ignorer, autrement dit, la parenté sonore des mots étalés sur la page pour exprimer cette retenue imposée?

Faut que je chuchote en clavardant, mes enfants, faut pas que je tousse, faut pas que je recule ma chaise de telle façon qu'elle érafle, sonore, la marquetterie, bref faut que j'éteigne mon cellulaire et toutes les cellules tonitruantes en moi, de même que les murmuratrices, tout ce qui trahirait mon infraction aux aimeux de la police. (172-173)

Alors qu'elle est souvent mise en ombre par la figure du chroniqueur du quotidien, observateur rêveur ou curieux sédentaire - «[s]poradiquement, j'arpente mon territoire sans bouger de mon bureau, en consultant MontréalCAM » (78) -, l'image de l'écrivain au clavier, en tête à tête avec la machine, n'échappe pas au lecteur. On peut ainsi noter, par exemple, un rapport sous le signe de l'efficacité avec la " nouvelle machine à écrire électrique » (145) qui possède non seulement une capacité à stocker et organiser l'information, mais aussi un incontournable logiciel de traitement de texte: "[r]etrouvé mon vieux fichier bibliographique planqué chez Geocities »; "[m]is en forme et livré le texte définitif pour Moebious 94 » (14). On trouve également une remarque sur un rendement plus bas de ce duo, remarque qui puise encore une fois dans le langage informatique: "[a]jouté une page à Origines. Les feuilles mortes se ramassent peut-être à la pelle, mais la mémoire vive, c'est un octet à la fois » (23). Certes, la manière dont ce rapport est mis en avant par l'auteur n'est pas toujours loin du lieu commun, mais, qu'il soit humanisé ou figuré comme bête, l'ordinateur s'avère indispensable dans le travail d'écriture dont Vacuum fait état. Ci et là, le scripteur consigne les hauts et les bas de cette relation avec son ordinateur, Memory Babe, - plus attachant que « la vieille Underwood » (84) -, tantôt pour expliquer sa complicité à la naissance du poème Fatalis, écrit en état de grâce, bien avant l'aventure du journal, tantôt pour avouer que cette magie n'a pas duré : " [s]i je peux ranimer mon enthousiasme pour cette machine et ses possibilités, je gagnerai encore quelques heures »(128). La symbiose entre les deux est telle qu'on ne peut pas ignorer, par exemple, l'exaspération de l'écrivain devant le comportement de sa machine devenue bête vorace dans un moment critique : "[q]uand mon ordinateur m'a mangé deux mille mots tantôt, je l'ai suspendu au-dessus du grand canyon de ma ruelle, décidé à en finir avec ce méchant vieux bestiau emphysémateux ॥ (157). Pour rendre compte de ces détails, les notations journalières puisent d'ailleurs dans un répertoire expressif impressionnant; il arrive peu souvent que le lecteur assiste à la manipulation d'un " clavier tout neuf, net, sec et nerveux " (128) ou qu'il soit avisé du dysfonctionnement d'un dispositif - «Ma souris m'a lâché. Celle en plastique et caoutchouc » (93) - sans que l'état d'âme de l'utilisateur soit prononcé.

Nous pourrions ajouter à ces considérations sur la représentation de l'univers mistralien un autre élément susceptible d'éclairer le rapport aux objets, à savoir la présence de l'ordinateur comme objet incitatif « evocative object », selon la formule de Sherry Turkle (11). Rappelons, dans le sillage de la chercheuse américaine, que, depuis le tournant du siècle, l'ordinateur est ce nouvel objet-miroir dans lequel tout un chacun se regarde différemment. II n'y a pas lieu de contester son pouvoir d'imprégner l'imaginaire de ceux qui se mettent devant son écran: la métaphore de l'ordinateur s'empare des questions que l'utilisateur se pose sur le monde et de la perception que celui-ci se fait de lui-même. II en est ainsi, on s'en doute, chez Mistral diariste. Ainsi, un 30 juin celui-ci s'identifie à une " méchante machine métisse " (108). Ailleurs, il choisit aussi de dénouer l'émotion ressentie lors des " retournements » de sa muse par une esquive sémantique sous le signe de l'informatique : 
Terreur de glace, c'est ce que j'ai trouvé de mieux pour dessiner mon sentiment, mais ce n'est pas, hein, tant s'en faut, une explication très porteuse de sens et de nuances ; c'est une approximation codée au moyen d'un langage commun dans le dessein de transférer l'information d'une mémoire à une autre, et c'est là un domaine où les humains sont désespérément moins efficaces que les ordinateurs. (104)

Dans une autre entrée, le diariste fait une blague en exploitant la valeur néologique du mot moniteur: " [l]e moniteur a le disc si dur qu'il est sur le point de télécharger sur mon bureau » (46). II ne manque pas non plus les lamentations sur la périssabilité de l'humain et l'inexorable passage du temps qui « s'occupe activement de notre pomme, la repeint, l'actualise, la met à jour comme une page web » (133). Rarement, l'ordinateur est cet autre inintelligible - « je cherchais le code de l'accent grave sur ce clavier anglais »qui accentue le sentiment de dépaysement : «[p]rivé d'ordinateur, je ne pourrai probablement pas alimenter le Journal autant que je le souhaiterais » (167).

\section{« De béants jours livrés à tous les regards » : l'écrivain et le processus d'auto- publication}

Ayant examiné la relation de l'écrivain avec son ordinateur telle qu'elle ressort des notations journalières de Vacuum, nous ne pouvons pas négliger les indices textuels qui renvoient au processus de mise en ligne du journal, processus dans lequel l'ordinateur fait partie d'un dispositif numérique d'envergure indispensable à l'auto-publication. II serait d'ailleurs utile de rappeler ici que l'auto-publication, facilitée par l'avènement des technologies de l'information et de communication, marquait un tournant, il y a plus de trois décennies, dans la façon d'aborder les écritures du moi et de penser leur rapport au lecteur. II en est ainsi du journal, cette écriture qui « sculpte la vie en direct » (Lejeune, "Composer un journal » s.p.) et qui fait depuis l'objet de nombreuses recherches, d'autant plus que le genre a connu un essor sans précédent.

Considéré pendant longtemps une forme d'écriture mineure, banale, informelle, destinée à soi-même (un objet de l'enfance qui recueille des propos souvent naïfs, un ami de papier à qui on raconte ses premiers émois amoureux ou un compagnon pour traverser un temps de crise à l'âge adulte), le journal personnel est, de nos jours, une pratique de masse dont la définition s'élargit. Pour les spécialistes du genre, soucieux d'en trouver le plus grand dénominateur commun, ce qui caractérise avant tout un journal support où l'on consigne ses pensées ${ }^{5}$ - c'est l'inscription du temps. Au-delà de la datation, geste qui renvoie à la mémoire et qui est censé donner au journal valeur de vérité, c'est le rapport au destinataire qui oriente les recherches sur cette forme d'écriture intime. Rappelons, avec Anthony Purdy, que l'intime ${ }^{6}$ « est l'espace d'un rapport, d'un échange, espace communautaire qui ne s'ouvre que grâce à l'image médiatrice d'un destinataire (ou d'une destinataire) transcendant(e) »(1). Internet, lieu d'échange par excellence, "permet de combiner intimité et publication », comme le souligne, sur le site " Autopacte », un Philippe Lejeune préoccupé par l'idée d'une anthologie des journaux («Une anthologie des journaux »). En lien avec ces deux propos, on pourrait également évoquer une célèbre phrase d'Ulysse gramophone qui permettrait d'esquisser une parenté entre le journal et la lettre ou encore la carte postale qui, elles, désignent explicitement un lecteur : " [t]oute écriture publique, tout texte ouvert est aussi offert comme la surface exhibée, non privée, d'une lettre ouverte, donc d'une carte postale, avec son adresse incorporée dans le message, dès lors douteuse, avec son langage à la fois codé et stéréotypé » (Derrida $63)$.

Notre lecture de Vacuum ne saura pas négliger la situation illocutoire particulière de ce journal : il émerge sur le web, grâce au dispositif informatique. C'est en témoin d'une réussite technique que le lecteur est d'ailleurs appelé à se pencher sur la première page du journal où se trouve inscrite la date du « 31.03 .02 » (13), soit un jour de Pâques. En ce jour de printemps et de résurrection, le diariste consigne comme suit la mise au monde de son cyberjournal :

Sué toute la sainte journée pour maîtriser Blogger, ce canasson rétif : le mettre en ligne en me concentrant sur le processus, et surtout pas sur le contenu, puisque somme toute j'ai toujours été contre le journal comme genre (cette idée de dire la vérité ! D'écrire sa vie pendant qu'on la vit!). Mais voilà, on dirait bien que ça marche, comme si j'avais percé de vastes apertures dans les murs et le plafond de mon studio, de béants jours livrés à tous les regards, et que je me tenais là, fier, si fier de savoir me servir d'un marteau-pilon...

Cette image du studio mistralien ouvert à tous les regards par l'entremise du blog évoque évidemment l'appétit pour la communication virtuelle qui caractérise notre monde et cette tendance contemporaine à diffuser l'intime à travers les cyber-supports'. Si l'on croit Annabelle Klein, le blog, plus que tout autre dispositif d'autopublication sur Internet « serait davantage un 'chez nous', un 'entre nous' où se trouve 
Buglea, Diana. « 'Vous êtes toujours là ? Ma souris m'a lâché' : cris, chuchotements et objets de l'écrivain dans le roman Vacuum de Christian Mistral. » Nouvelle Revue Synergies Canada, №13 (2020)

accentuée la dimension d'ouverture à l'autre en intégrant dans ce dispositif d'énonciation les multiples points de vue » (159).

Notons, en même temps que le geste d'apprivoisement de l'outil numérique, "ce canasson rétif ", les propos d'une instance écrivante réfractaire à la règle de dire la vérité ${ }^{8}$ propre à toute démarche autobiographique. Notons aussi que celui qui dit je se rebiffe contre l'idée d'« écrire sa vie pendant qu'on la vit ». La notation journalière est éphémère, sans portée : il ne faut "surtout pas [se concentrer] sur le contenu »- le pacte de lecture est ainsi scellé, dès le début. Au fur et à mesure que le journal avance, sa visée pragmatique se dessine davantage, alors que les doutes sur le choix du genre persistent :

Ce journal, c'est autre chose. Longtemps je me suis refusé, incapable de substituer l'acte d'écrire à celui de vivre, certain que le processus ne pouvait déboucher que sur l'absurde. De plus, je tenais ce genre pour particulier en ce qu'il s'adressait à des lecteurs futurs; or cela me créait une obligation de dépeindre la vérité, toute ma vérité, un exercice aussi périlleux que futile auquel je répugnais à me soumettre. Mais le fait de tenir un journal pour des lecteurs immédiats change la donne, à mon sens : c'est le vrai contre la vérité sélective, ou ce qu'on appelle aujourd'hui avec quelque abus l'autofiction. De plus, la seule perspective d'être lu et d'en jeter plein la vue me donne l'impulsion nécessaire à l'ouvrage quotidien. C'est comme ça. II est de pires raisons d'écrire. (Vacuum 64)

C'est donc en tant qu'objet de lecture que le journal acquiert sa raison d'être. Aussi la réussite technique, le mettre en ligne, est-elle importante, assurant ce contact immédiat avec l'Autre et donc une plus-value à l'aventure d'écriture quotidienne. L'impact sur les « lecteurs immédiats » est d'ailleurs visible en chiffres de fréquentation, au grand bonheur du diariste : "[l]es outils de mesure de fréquentation propres au web n'ont pas encore d'équivalent dans l'édition traditionnelle » (107). On apprend vite que « la fréquentation du site a augmenté de $59,1 \%$ depuis la publication du Journal, soit une dizaine de jours » (20), pour voir plus loin « ce Journal-Toile » réclamer « dix mille visiteurs » (205). Les statistiques réconfortent : " [c]e Journal a une douzaine de semaines et son lectorat augmente de façon régulière, sans sursauts suspects, suivant une courbe qui suggère que nous avons affaire à du solide » (107). La question des éphémérides ainsi réglée, c'est la superstition qui plane quand le compteur du journal affiche 666, poussant le diariste à s'engager à « faire un croche par le bénitier » (33).

Nous saurons sans doute remarquer qu'au-delà des chiffres et sans toujours arborer une posture provocatrice ou cocasse, le sujet peut simplement évoquer l'autre sous un mode phatique : " [v]ous là-bas dans l'ombre, et vous ici, dans la lumière des premières rangées, je vous aime bien, vous savez... " (95). Maître des cérémonies, il appellera au rendez-vous ou congédiera à son gré : « [h] ey, l'm back ! (95); « That's it folks. Go home to your loved ones. Show's over for now » (113). Parfois, le lecteur est invité à moissonner les nouvelles plus tard, la notation du jour étant suspendue alors que se formule un souci quant à la mise en forme :

Salut la compagnie. J'écris pour énumérer brièvement ce sur quoi je n'écrirai pas maintenant, et pourquoi. Je n'ai pas fermé l'œil depuis cinquante heures, voilà pourquoi. Lessivé, je n'écris pas plus de conneries que d'habitude, mais je les écris moins bien. Alors, à plus tard, hein ? (101)

Peu importe le registre que le diariste choisit pour livrer cette parole personnelle, à l'horizon de l'écriture, il y a toujours l'autre, présence réelle ou regard dans l'ombre, complice du destin du journal ou lecteur distant. Le monde est laid, soupire parfois le diariste qui trouve alors refuge dans l'espace lumineux du dictionnaire.

\section{Le dictionnaire, trésor des mots pour l'écrivain ${ }^{9}$}

Alors que l'ordinateur est ce dispositif incontournable qui a remplacé la machine à écrire et qui prend le plus de place parmi les objets et instruments de la boite à vivre mistralienne, le dictionnaire semble être un objet plus discret, mais pas du tout négligeable dans le processus d'écriture. Dans ses entrevues, Mistral avoue d'ailleurs son amour du dictionnaire ${ }^{10}$, son penchant à dénicher les mots, visible dans le journal luimême. Cependant, le diariste ne se contente pas de nous faire part des mots qui l'intriguent ou l'enchantent, il les invite à s'expatrier en toute splendeur sémantique pour mieux les accueillir dans son texte. Insérés dans l'espace textuel de Vacuum comme entités lexicologiques intactes, les mots sont enrobés de commentaires censés les assortir à l'univers que l'on connaît. Pour illustrer les « mots du jour » du 24 avril, la plume se fait, comme tant de fois, cocasse :

- épistaxis, n.f. Saignement de nez. Comme dans J'te mens pas, bonhomme, j'ai coké jusqu'à épistaxis. 
Buglea, Diana. « 'Vous êtes toujours là ? Ma souris m'a lâché' : cris, chuchotements et objets de l'écrivain dans le roman Vacuum de Christian Mistral. » Nouvelle Revue Synergies Canada, №13 (2020)

- hébéphrénie, n.f. Forme de démence précoce. Psychose considérée comme une forme de schizophrénie. Comme dans Max Cockrell est atteint d'hébéphrénie en plus de se prendre pour un autre. (31)

Ailleurs, le mouvement est inversé : c'est la définition de dictionnaire qui vient contaminer le discours du diariste et l'émotion que celui-ci ressent. Alors même qu'il fustige les aberrations d'un système social pervers où les individus sont laissés à eux-mêmes, souvent sans espoir, tels les étudiants décrocheurs dont le diariste déplore le destin, une parenthèse s'ouvre pour fournir le plein sens de l'expression " prendre la tangente » (232), avec ce qu'elle comporte d'esquive, de faux-fuyant. II arrive aussi qu'une logique poétique préside à la mise en mots de soi ou du monde. Ainsi, un jugement sur soi peut s'accompagner d'un mouvement définitionnel digne de l'imaginaire surréaliste. "Mon cœur est un chien roux qui ronge une mâchoire de mouton à l'ombre des palétuviers " (228), nous révèle la seule entrée de la journée du 7 décembre, alors que le lendemain, il s'agit d'un « pain de plastic dissimulé sous un gibus calamistré » (229). Parfois, les définitions constituent simplement une prise de parole ludique, comme il ressort de la notation du 15 août. "Sinesthétique, ou l'esthétique du péché ", se plaît alors à consigner le diariste, prêt à se réclamer d'une nouvelle école littéraire (154). Un même principe met au clair la signification de Vortex Violet. Le titre racoleur du cycle romanesque mistralien est ainsi décodé, allitération à l'appui : « en gros, c'est le violent velouté vagin virtuel de la Vie » (152).

Le travail sur la matérialité de la langue est également visible au niveau de la ponctuation, de l'orthographe et de la typographie. Ainsi, au début de la première entrée du 18 mai, les mots s'enchaînent insoumis à la ponctuation - ne dit-on d'ailleurs qu'écrire c'est parler sans être interrompu - unis dans une sorte de verbiage triomphant dont les mots saillants pourraient être "les marais de souffrance " autant que les " effets de manche ", "l'avenir écrasant somptueux» ou tout autre (57). Ailleurs, c'est l'orthographe qui est déstabilisée, souvent par une parole qui la rapproche de la transcription phonétique "KwAAA ? » (20), "Keskia » (126) - formes langagières qui se répandent depuis l'avènement des nouvelles technologies de communication. «Tu veux dire que ces busards se précipitent maintenant que tu tiens UN JOURNAL ? » (20), commente un proche intrigué par l'intérêt du public pour ces " trivialités » - comme un autre personnage qualifie sa propre pratique journalière. La typographie traduit cet étonnement, sinon ce mépris, tout en attirant l'attention sur l'essence de Vacuum, sa nature, son ancrage générique.

\section{«Ce journal dont je veux faire un livre »}

Dans le texte qui nous occupe, l'ouverture à l'autre, réglée par le fait même de la publication en ligne, non seulement aidera à faire avancer la notation diariste - «la perspective d'être lu et d'en jeter plein la vue » (64) constitue d'ailleurs le moteur de l'écriture -, mais aussi, comme nous allons le voir, affectera le destin du journal. L'idée même d'en faire un livre surgit lors d'une conversation avec un ami, lecteur à son tour du cyberjournal. " C'est vrai ! Tu le publies déjà sur le Net, au fur et à mesure, ajoutera un autre, et puis t'en as jamais fait mystère : un écrivain écrit pour publier » (39). Alors que se profile à l'horizon un possible anoblissement éditorial, le diariste renchérit l'idée euphorique de l'autre : « [o]n pourrait croire que je perds jamais de vue l'objectif de le publier » (39), d'autant plus qu'au fil des jours se multiplient les moments de détresse où les mots ne viennent pas s'aligner " dociles, comme des régiments de zouaves pontificaux » (160), pour faire aboutir le projet d'Origines - livre en chantier dont l'écriture accuse un manque de souffle. Tout au long du journal, le corps morcelé de ce livre nous est révélé de façon indirecte, à travers la cadence inégale du travail d'écriture qui alimente ce manuscrit d'un paragraphe, d'une page ou d'une quantité de mots indéterminée: «[a]jouté tant soit peu à Origines. Me demande si ce n'est pas le tremblement de terre à 6:42 ce matin qui m'a remué les puces » (27). On apprend que l'idée obsessive de cette prose remonte à l'adolescence et se trouve une parenté avec les ouvrages issus d'un même désir d'investigation généalogique à la fin des années soixante-dix :

J'avais quatorze ans quand j'ai conçu le projet d'un grand livre, un cycle sur les sources de la famille, en commençant avec Marin Boucher et qui descendrait jusqu'à Hector. C'était dans la mouvance de Racines, qui déclencha la folie des recherches généalogiques et j'avais choisi comme titre Origines, et je me croyais très fûté. (Vacuum 121)

Tandis que les pages d'Origines, livrées " au compte-gouttes » (64), sont souvent mises en question, le diariste reconsidère "l'agrégat géologique » (181) de son cyberjournal qu'il a l'idée de transformer en livre le 28 juillet : " [s]ongé à détacher ce Journal d'Origines pour en faire au bout d'un an le quatrième tome de Vortex Violet: VACUUM » (Vacuum 134). Par une sorte de construction en abyme, nous assistons plus tard à sa naissance: "[a]vec $\mathrm{K}$, on a imprimé les 125 premières pages, y compris les exergues, la dédicace, le faux-titre et une couverture en couleur. Me suis amusé comme un débutant » (181). Prothèse de l'œuvre à venir (comme on a pu le montrer de la lettre chez quelques grands écrivains du XIXe siècle 
Buglea, Diana. « 'Vous êtes toujours là ? Ma souris m'a lâché' : cris, chuchotements et objets de l'écrivain dans le roman Vacuum de Christian Mistral. » Nouvelle Revue Synergies Canada, №13 (2020)

qui en ont fait un "laboratoire d'écriture ", une matrice de création), le journal mistralien est d'ailleurs parsemé de tout un éventail de manœuvres diégétiques et metatextuelles: «[d]ans ce journal, dans ce roman, j'énoue l'étoffe rêche d'une existence américaine, la mienne ; j'époutis son tissu et j'épince sa trame, j'aspire à vide, je vacuume » (Vacuum 198), se confie le sujet, mettant ainsi le signe d'égalité entre l'écriture du jour et la création romanesque. Si le dernier jour de l'an, le diariste a " tout un tas de libertés d'expression à comprimer en quelques phrases » (253), un mois avant, il admettait l'" erreur de concevoir un roman comme une auberge espagnole, avec de tout pour tous " (197). A son tour lecteur d'autres journaux en ligne - dont notamment celui de sa bien-aimée qui le quitte -, le sujet écrivant se trouve confronté au dilemme qu'éprouve le lecteur de Vacuum : " je ne distingue plus la fable du vraisemblable » (190). Plus on approche la fin du livre, plus les fragments aspirent à faire un tout - ne dit-on souvent à propos de la pratique diariste que chaque fragment est un ensemble vivant, une cellule qui figure l'organisation, "les pulsions de la totalité » (Didier 245)? Le début et la fin du livre se répondent d'ailleurs : fête de Pâques en ouverture, fête de Noël neuf mois après, la même chemise noire pour les retrouvailles en famille, le bouton « recousu » (246) en vue de l'événement. "Assis en bout de table à nouveau, note le sujet, (il semble que j'aie finalement mérité cette place, en l'absence de mon père), cette fois, je n'ai pas souffert de la chaleur, car le soleil semblait froid » (247). La boucle se ferme, le tissu textuel est cousu pour qu'il tienne ; le journal qui s'écrit du 31 mars au 31 décembre se donne à lire, trois jours avant la fin de l'année 2002, comme un parcours réfléchi, mais émouvant :

Pourtant je m'y suis mis quasiment chaque jour, et plusieurs fois encore. A mon gré, sans jamais avoir l'impression de travailler, conscient pourtant du fil suivi et de la forme désirée. Mentant le moins possible et toujours pour la bonne cause, n'omettant rien sciemment sans en faire mention. Et puis, petit à petit, cela m'apparaît maintenant, j'ai compris que ma prémisse était fausse, et que celui qui prend le temps de consigner par écrit les tenants de sa vie reçoit en retour le rare privilège d'en vivre une deuxième, contiguë. (249)

Ainsi voué à s'épanouir au sein d'une écriture expressive ${ }^{11}$, excessive, hautement émotionnelle, qui abrite des êtres et des objets investis d'une forte charge affective, le sujet écrivant se construit une identité intime mobile et ombrée, tout en restant fidèle au principe qui anime son ethos auctorial : " tanguer tout droit, grand fabulant, jusqu'au fond de ce soir » (254).

\section{Conclusion}

Journal personnel - parsemé de cris et chuchotements - qui n'échappe pas aux attraits de la fiction, Vacuum nous a permis de réfléchir à l'écrivain dans sa relation avec les objets qui l'entourent, notamment ses instruments de travail, à savoir l'ordinateur, Memory Babe, qui a détrôné la vieille machine à écrire, le dispositif blogueur, capricieux mais efficace, qui a permis l'auto-publication du texte, et le dictionnaire, trésor des mots qu'il chérit. Nous avons compris que ce trio instrumental s'est avéré indispensable au diariste pour rendre compte de sa musique intérieure, de son quotidien hanté par le projet d'un livre qui peinait à murir, de ce besoin de se dire, de se montrer aux autres - serein, euphorique ou houleux simplement humain.

\section{Notes}

${ }^{1}$ Souvent considéré comme instrument (objet) de manipulation, l'entretien permet à l'écrivain de gérer son image, d'orienter la réception de ses écrits. Voir en ce sens l'article de Karin Schwerdtner et de Geneviève de Viveiros, « Au risque du métatexte. Formes et enjeux de l'autocommentaire littéraire ».

${ }^{2}$ Christian Mistral a également publié Cockrell dehors dedans (1989), Papier-mâché (1989), Fatalis (1992), Julien Vago (1993), Carton-pâte (1995), Sylvia au bout du rouleau ivre (2001), Origines (2003) et Léon, Coco et Mulligan (2007).

${ }^{3}$ De nombreux chercheurs dont Sherry Turkle ont conclu que derrière le souci de ce qui se passe sur l'écran, c'est l'intérêt pour ce qui se passe en soi-même que l'utilisateur de l'ordinateur manifeste.

${ }^{4}$ Bien souvent, l'écrivain contemporain remplit non seulement son rôle d'auteur, mais devient aussi, dans le cas d'un texte publié en autoédition, éditeur, informaticien, blogueur... 
Buglea, Diana. « 'Vous êtes toujours là ? Ma souris m'a lâché' : cris, chuchotements et objets de l'écrivain dans le roman Vacuum de Christian Mistral. » Nouvelle Revue Synergies Canada, N¹3 (2020)

${ }^{5}$ Également retenus comme traits caractéristiques du genre, la forme ouverte, la fragmentation, la consignation des faits à vif situent le journal à l'antipode de l'autobiographie qui suppose un recul, un regard rétrospectif et une position de surplomb adoptée par le narrateur qui rend compte de lui-même au passé dans une démarche totalisante des faits remémorés.

${ }^{6}$ Pour les dictionnaristes, l'intime, associé à l'intériorité, au retrait, à l'indicible, à l'incommunicable, renvoie à ce qu'il y a de plus profond dans chaque être, ce qui fonde sa singularité. Cependant, le terme dépasse la sphère affective, cumulant des notations d'une grande diversité. Les travaux de l'universitaire suisse Dominique Kunz Westerhoff consacrés au journal intime s'attachent, entre autres, à cette question.

${ }^{7}$ Ces pratiques d'écriture relèvent d'ailleurs d'une tendance généralisée du monde occidental à la surexposition de la vie intime que Serge Tisseron propose d'appeler "extimité ». Le livre de Sherry Turkle, Alone Together, analyse aussi notre relation ambivalente avec une technologie qui redéfinit notre perception de l'intimité et transforme le paysage de notre vie affective.

${ }^{8}$ Voir l'article d'Annie Cantin « Les écritures intimes aux frontières du réel ou une littérature du vrai est-elle possible?".

${ }^{9}$ L'essai de Gisèle Mathieu-Castellani, La rhétorique des passions, situe l'émotion, la passion, au cœur de l'entreprise de séduction qui anime aussi bien l'orateur que l'écrivain ou le poète.

${ }^{10}$ L'article d'Aline Francoeur, « L'écrivain français et le dictionnaire dans son œuvre : objectivation, symbolisation, symbiose constante ", fait état de la relation privilégiée que les écrivains entretiennent avec leur dictionnaire, objet indispensable pour leur travail d'écriture.

${ }^{11}$ Nous renvoyons au tout récent article de Karin Schwerdtner, «Présentation : entre public et privé : lettres d'écrivains depuis le $X I X^{e}$ siècle », qui souligne, dans un même sens, la préoccupation de l'écrivain épistolier pour la forme que prend la révélation de soi et le travail stylistique qui accompagne souvent ce genre d'écriture chez les gens de lettres.

\section{Bibliographie}

Cantin, Annie. "Les écritures intimes aux frontières du réel ou une littérature du vrai est-elle possible ? » Fabula, www.fabula.org/forum/colloque99/228.php. Consulté le 8 février 2019.

Derrida, Jacques. Ulysse gramophone. Deux mots pour Joyce. Galilée, 1987.

Didier, Béatrice. «Le lecteur du journal intime. » Actes du colloque des 14 et 16 juin 1984 à Reims, édité par Michel Picard, Clancier-Guénaud, 1987, pp. 229-255.

Francoeur, Aline. « L'écrivain français et le dictionnaire dans son œuvre : objectivation, symbolisation, symbiose constante ». Mémoires du livre, vol. 2, no. 2, printemps 2011, pp. 1-28. Érudit, https://id.erudit.org/iderudit/1001763ar. Consulté le 16 juillet 2019.

Klein, Annabelle. «Entre médiation et médiatisation, dynamiques identitaires en jeu dans les dispositifs d'autopublication sur Internet ». ESSACHESS, vol. 3, no. 6, 2010, pp. 147-162, www.essachess.com/index.php/jcs/article/view/10. Consulté le 30 janvier 2019.

Kunz Westerhoff, Dominique. « Le Journal intime. » Méthodes et problèmes. Université de Genève, 2005, https://www.unige.ch/lettres/framo/enseignements/methodes/journal/jiintegr.html. Consulté le 22 février 2019.

Lejeune, Philippe. « Composer un journal ». Lieux d'être, no. 30, 2000, pp. 151-155, www.autopacte.org/Composer_un_journal.html. Consulté le 27 février 2019.

---. « Une anthologie des journaux : pourquoi et comment ? ». Autopacte, 2006, www.autopacte.org/AnthologieCordoue.html. Consulté le 28 juin 2019. 
Buglea, Diana. « 'Vous êtes toujours là ? Ma souris m'a lâché' : cris, chuchotements et objets de l'écrivain dans le roman Vacuum de Christian Mistral. » Nouvelle Revue Synergies Canada, N¹3 (2020)

Mathieu-Castellani, Gisèle. La rhétorique des passions. PUF, 2000.

Mistral, Christian. Fatalis. XYZ, 1992.

---. Fontes - poèmes et chansons, 1985-2003. Triptyque, 2004.

---. « Présentation ». Christian Mistral. http://christianmistral.com/pre.html. Consulté le 20 janvier 2019.

---. Vacuum. Trait d'union, 2003.

---. Valium. XYZ, Romanichels, 2000.

---. Vamp. Québec/Amérique, 1988.

---. Vautour. XYZ, 1990.

Purdy, Anthony. « Stratégies de l'intime : pour une théorie pragmatique de la communication stendhalienne. » Les œuvres intimes, édité par Jean-Jacques Hamm, Queen's University, 1997, pp. 1 47 .

Schwerdtner, Karin. «Présentation : entre public et privé : lettres d'écrivains depuis le $\mathrm{XIX}$ siècle. » Études françaises, vol. 55, no. 1, 2019, pp. 5-15. Érudit, https://id.erudit.org/iderudit/1059364ar. Consulté le 12 juillet 2019.

Schwerdtner, Karin et Geneviève de Viveiros. « Au risque du métatexte. Formes et enjeux de l'autocommentaire littéraire. ॥ Interférences littéraires / Literaire interferenties, no. 15, février 2015, pp. 7-15, http://www.interferenceslitteraires.be/index.php/illi/article/view/225/156. Consulté le 20 juillet 2019.

Tisseron, Serge. L'intimité surexposée. Ramsay, 2001.

Turkle, Sherry. Alone Together: Why We Expect More from Technology and Less from Each Other. Basic Books, 2011.

---. The Second Self. Simon and Schuster, 1984. 